to snow-blindness is really caused, certainly in part, by desiccation and perhaps also freezing of the corneal epithelium, and not by excessive ultra-violet light reflections.

(5) The condition could be prevented by the wearing of gogglés.

(6) The name desiccation keratitis is suggested for the condition.

I would like to express my gratitude to Brigadier C. W. Graham and Brigadier G. I. Scott and to Dr. Rowland Wilson, of the Giza Memorial Hospital, Cairo, for their great help and advice in the preparation of this paper, and to Colonel $\mathrm{J}$. Morrison, O.B.E., M.C., for permission to publish it.

\title{
THE SECRETION-DIFFUSION THEORY OF INTRA-OCULAR FLUID DYNAMICS*
}

BY

\author{
V. Everet T Kinsey and W. Morton Grant \\ HOWE LABORATORY OF OPHTHALMOLOGY, HARVARD UNIVERSITY \\ MEDICAL SCHOOL, BOSTON, MASSACHUSETTS
}

Recentiy Duke-Elder and .Davson ${ }^{1}$ discussed certain phases of our paper entitled " The mechanism of aqueous humour formation inferred from chemical studies on blood-aqueous humour dynamics" 2. From their comments it is apparent that these authors obtained the wrong impression concerning our viewpoint, Perhaps as a consequence they incorrectly modified certain equations which we had set up to represent the ultra-filtration mechanism of aqueous humour formation. We feel therefore that some recapitulation of our theory of intra-ocular fluid dynamics is in order. At the same time we wish to discuss the criticisms which DukeElder and Davson have made concerning the mathematical concepts we have used in developing our theory.

Through the use of heavy water $\left(\mathrm{D}_{2} \mathrm{O}\right)$ as a tracer substance for ordinary water it was shown that one half of the water in the anterior chamber of the rabbit was replaced in slightly less than three minutes ${ }^{3}$. This is equal to a total water movement into and out of the anterior chamber of approximately fifty cubic millimetres per minute, a rate, it will be observed, far in excess of that which was thought to correspond to the rate of formation of. aqueous humour as a whole. This finding suggested that the various constituents of the aqueous humour may enter the anterior chamber at different rates, and that it was unrealistic therefore, to speak of a rate of formation of aqueous humour as a whole. To

* Received for publication, February 4, 1944. 
test this hypothesis the rates of accumulation of sodium and of chloride in the anterior chamber were measured under strictly physiologic conditions by employing radio active isotopes of these elements. ${ }^{4}$ From the rate of accumulation of these ions it appeared that their rate of entry from the blood was equivalent to approximately four cubic millimetres of whole aqueous humour per minute. From the difference in rate of movement of water and these two ions it appeared certain that in the formation of aqueous humour all the constituents do not enter at a common rate, i.e., " as a whole," but that they are transferred from blood to aqueous humour at rates which are characteristic for each substance.

In order to test this idea further, the rates of accumulation in the aqueous humour of various other substances were determined $^{5}$; these included the ions thiocyanate, bromide, lithium and phosphate, and the non-electrolytes, urea and levulose. A record of the concentrations of these substances in the plasma was obtained and the concentration in the aqueous humour was determined and related to the maximum diffusible concentration in the plasma of each animal.

Having obtained these experimental data we then set out ${ }^{2}$ to see whether they were consistent with what would be expected on the basis of the following postulated mechanisms. First possibility : constituents of aqueous humour separately may enter the anterior chamber from the blood by a process of ultra-filtration (diffusion) where, under static conditions, they would accumulate until the concentration in the aqueous reached that in the blood. Second possibility: they enter by a process of ultra-filtration but leave by another mechanism, e.g., flow. Third possibility : they enter by a secretory process, in which instance it is necessary that there be some leakage (flow) out of the anterior chamber. It was pointed out that some combination of these processes (or others) might actually be involved in aqueous humour formation.

Equations consistent with the above possible mechanisms were formulated relating rates of transfer of a substance into and out of the anterior chamber to the ratio of its concentration in the aqueous to that in the blood at various intervals after its introduction into the blood.

.Then for each substance tested a determination was made of the best fit obtainable on the basis of each of the above assumptions for every experimentally determined concentration in the aqueous humour. This fit was determined by plotting the rate of transfer into the aqueous humour against a series of assumed rates of transfer out of this chamber for all of the experimentally found concentration ratios on the basis of both the ultra-filtration and secretory mechanisms. This method of treatment gave rise to two sets of values, one set for each mechanism. 
It was found that all the experimental data agreed well with an assumed rate of leakage out of the anterior chamber of approximately four cubic millimetres per minute. Thus, of the total water movement (fifty cubic millimetres per minute) between the blood and aqueous humour, only about one-twelfth could be accounted for on the basis of a through and through circulation; the remainder appears to be simply exchanged between blood and aqueous. It was found, furthermore, that the data resulting from tests made with the electrolytes were compatible only with the idea that all of these substances entered the anterior chamber as a result of a secretory process. While it could not be decided on the basis of these experiments whether the non-electrolytes enter the anterior chamber by ultra-filtration or secretion, it is our opinion, based to a considerable extent on the experimental studies of others, that non-electrolytes enter the anterior chamber by ultra-filtration. Because of our lack of exact knowledge concerning the pressure relationships it would appear better to substitute the more general term diffusion for the term ultra-filtration.

Thus we can summarize our present concept of aqueous humour dynamics by stating our belief that the electrolytes enter the anterior chamber as a result of secretion; the non-electrolytes enter by diffusion. Both electrolytes and non-electrolytes, we believe, leave the anterior chamber by a process of flow.

The process may be pictured as follows : the electrolytes are transferred as a result of a secretory process from the blood to the aqueous humour across a barrier which appears to be essentially impermeable to the simple diffusion of ions. The nature of the mechanism by which such secretion may take place has been discussed by Friedenwald. 6 The electrolytes are secreted at a rate which is apparently sufficient to maintain their concentration in the aqueous humour slightly in excess of that in the blood-so that the total osmotic activity is equivalent to about $5.4 \mathrm{~m}$.M. of sodium chloride per kilogram of water higher than in the blood?. This hypertonicity accounts for the continual passage of water into the eye, and also, we believe, for the intra-ocular pressure. The magnitude of the pressure is normally limited by the continuous loss of fluid by outflow from the anterior chamber.*

The non-electrolytes, like urea and glucose, enter the eye in a manner similar to water, i.e., by diffusion, and do so at a relatively slower rate than water. Accordingly, since there is an outflow of aqueous humour the concentration of non-electrolytes is normally less than in the blood. This may be seen more readily perhaps by considering the following hypothetical case.

- Were there no outflow, the difference in osmotic activity is sufficient to produce a pressure in an otherwise normal eye of about 165 millimetres of mercury. Any increase in the permeability of the blood-aqueous barrier results in lowered intraocular pressure due to abolition of the osmotic pressure difference. 
Accepting the rate of leakage out of the anterior chamber of four cubic millimetres per minute, let us consider a substance (nonelectrolyte) which exists in the blood in a concentration of 0.01 milligram per cubic millimetre, i.e., one part per hundred. Suppose that this substance has such properties with respect to the blood-aqueous barrier that it enters the anterior chamber at a rate of 0.005 milligram per minute, that is to say, one part per eight hundred of water. (It is, of course, because of the hypertonicity of the aqueous humour that it is possible for water to enter the eye disproportionately faster than the non-electrolytes). It may readily be calculated from equation $(1$ a) or $(4)$ which appear in a following section that under these conditions the concentration of the substance in the aqueous humour will be only 11 per cent. of that in the blood. Were the rate of entrance of the substance still slower relative to water, as would be the case for a larger molecule, or if the rate of flow out of the anterior chamber were more rapid, the concentration of the hypothetical substance in the anterior chamber would be still less.

Duke-Elder and Davson, in presenting their criticism, apparently failed to distinguish our consideration of the various possible mechanisms from our subsequent conclusions as to which, if any, of these mechanisms were in agreement with our experimental data. They limited their discussion to one of the mechanisms which we had considered, the one consisting only of ultra-filtration and outflow, and claimed that such a simple mechanism is improbable because of the energy requirements. Apparently they did not appreciate that such a mechanism was considered only in order to determine how it compared with the other mechanisms in accounting for the observed behaviour of individual test substances. They cite the mathematical development which we present to make this determination*, and state (page 433) that we are in error in our argument because we integrate equation $(1 \mathrm{a})$ at a constant volume. On the contrary, the integration of equation $(1 \mathrm{a})$ at a constant volume is valid since there is no question of any change in the volume in the eye. Duke-Elder and Davson introduce a replacement concept which is
*(1) $\frac{\mathrm{dA}}{\mathrm{dt}}=\mathrm{k}_{2}\left(\mathrm{C}_{1}-\mathrm{C}_{2}\right)$
$\mathrm{C}_{1}$ equals concentration in the blood.
$+\mathrm{C}_{2}$ equal concentration in the aqueous humour at any time.
(1a) $\mathrm{dA}=\mathrm{k}_{2}\left(\mathrm{C}_{1}-\mathrm{C}_{2}\right)-\mathrm{k}^{\prime} \mathrm{C}_{2}$ (where $\mathrm{A}$ equals the amount of constituent $\frac{\mathrm{dA}}{\mathrm{dt}}$ when $t=\infty$ )
(4) $\underset{\mathrm{C}_{1}}{\mathrm{C}_{1}}=\frac{\mathbf{k}_{2}}{\mathbf{k}_{2}-\mathbf{k}^{\prime}}$ present in aqueous humour).

+ Duke-Elder and Davson properly call attention to our original somewhat confusing notation. $C_{2}$ should equal concentration in aqueous humour at any time instead of at a given time, $t_{1}$. 
based on the argument that the condition. of constant volume requires the addition of "fluid" (containing the substance in question) in amounts over and above that which is consistent with 'equation (1), i.e., than may be accounted for on the basis of the ultra-filtration process. It is our contention that such an argument is fallacious. Their equation (5)* which, like our equations, deals only with a constituent and not with fluid, states that an additional amount of this substance will enter the anterior chamber by a process different from that which we had postulated, viz., one of ultra-filtration, wherein by definition, the rate of change of $A$ is entirely dependent upon the difference in concentration of a constituent between blood and aqueous humour. They believe the constituent will enter in addition by a process described by the factor $k^{\prime} C_{1}$. It is clear that such a hypothetical process is completely different from one of ultra-filtration which was originally postulated.

Now with regard to the replacement of fluid lost from the anterior chamber, we already discussed this problem in connection with these particular equations and said " moreover, it is assumed that the relative ease with which water may move across the barrier in either direction, as shown in ${ }^{3}$, permits the volume, hence the intra-ocular pressure, to remain essentially constant despite loss of fluid by means of flow."

That Duke-Elder and Davson's whole argument is invalid can perhaps be more readily visualized through the use of a simple model. Suppose we picture a tank (with infinite capacity to be replenished) filled with water containing suspended particles. To one side of this tank is joined a second smaller tank which is separated from the first by a screened partition which will permit the slow exchange of these particles and the rapid exchange of water between the two tanks. Attached to the smaller tank is a tap so that water and particles may be drained off at will. 'The particles will eventually distribute themselves evenly throughout the two tanks provided there are no leaks. If, however, the tap is opened and left open (analogous to flow out of the eye) the concentration of the particles in the second tank will decrease and when a steady state is reached the relative concentrations in the two tanks will clearly depend on the relative rate of passage of particles from the larger to the smaller and out of the latter.

Applying Duke-Elder and Davson's comments to our model they would say that if the volume in the smaller tank is to remain constant the fluid lost from this tank must be replaced by fluid from the first tank, and indeed this is true, but it will not "clearly increase the rate of increase of $A$, the amount of substance in the

$$
\frac{d A}{d t}=k_{2}\left(C-C_{2}\right)-k C_{2}+k^{\prime} C_{1} \text {. }
$$


aqueous" (second tank) " by a factor $\mathrm{k}^{\prime} \mathrm{C}_{1}$ ", . Moreover, it is not necessary to argue, as they suggest, that the fluid which replaces the aqueous húmour lost via Schlemm's canal is free from the particular (dissolved) substance. Referring again to our model the rate of entrance of the particles (assuming a diffusion process analogous to that in the eye) will be proportional to the difference in concentration in the two tanks just as we postulate in equation ( $1 \mathrm{a})$. The effect of an increase in the rate of outflow will be to lower the relative concentration in tank number two. This will increase the difference between $C_{1}$ and $C_{2}$, but it will not mean that the physics of the whole model will be changed so that, in addition to particles entering due to the assumed diffusion process, they would also enter at a rate proportional to the concentration in the first tank, as Duke-Elder and Davson stated would occur in the eye.

In the last paragraph of their paper Duke-Elder and Davson claim to restate their criticism for the benefit of the non-mathematical reader. They say that " if the concentration of a given substance, say urea, is higher in the blood than in the aqueous humour, the system plasma-aqueous humour, is not in equilibrium and hence diffusion of urea should take place into the aqueous humour until the equilibrium condition of equal concentrations in the two fluids is achieved." We agree with this statement provided there is no drainage. They then say that we claim that "as soon as drainage occurs the concentrations become different." Perhaps they would have expressed our view better had they said that " if we assume that there is drainage the concentrations never are equal." However, with the general idea, we appear to be in agreement. Duke-Elder and Davson then proceed with their argument by saying that " the second law of thermodynamics states that a system of this kind can only be maintained at a non-equilibrium position by the continued performance of work on it. Drainage of fluid away from the anterior chamber is not capable of performing the necessary work on the system " they say, "so . that in the absence of secretory activity the concentration differences claimed by Kinsey and Grant are excluded by the second law." We agree with their statement of the demands of the second law of thermodynamics, and also with their idea that the concentration difference which we found for non-electrolytes would be excluded by this law were there no secretory activity. Since we have repeatedly expressed the conclusion in our paper ${ }^{2}$ that " all the monovalent electrolytes tested entered the anterior chamber as a result of a secretory process,' it would appear that the argument, if indeed it be one, against our theory of intra-ocular fluid dynamics is not relevant. 


\title{
REFERENCES
}

1. Duke-Elder, S. and Davson, H. (1943).-Brit. Ji. Ophthal., Vol. XXVII, p. 431.

2. KInsey, V. E. and Grant, W. M. (1943).-Jl. of Gen. Physiol., Vol. XXVI, p. 131.

3. Kinsey, V. E., Grant, W. M. and Cogan, D. G. (1942)-Arch. of Ophthal., Vol. XXVII, p. 242.

4. Kinsey, V. E.. Grant, W. M., Cogan, D. G., Livingood, J. J. and Curtis, B. R. (1942). - Arch. of Ophthal., Vol. XXVII, p. 1126.

5. Kinsey, V. E. and Grant, W. M. (1942).-Jl. of Gen. Physiol., Vol. XXVI, p. 119.

6. Friedenwald, J. S. and Stiehler, R. D. (1938).-Arch. of Ophthal., Vol. XX, p. 761.

7. Roepke, R. R. and Hetherington, W. A. (1940).-Amer. Jl. of Physiol., Vol. CXXX, p. 340.

\section{THE LATE RESULTS OF REMOVAL OF INTRA- OCULAR FOREIGN BODIES WITH THE MAGNET *}

\author{
BY \\ Capt. P. D. TRevor-ROPER, N.Z.M.C., \\ LATE R.S.O., MOORFIELDS EYE HOSPITAL
}

THERE have been few recent publications of the late results of the removal of magnetic intra-ocular foreign bodies, so that a follow-up of the cases treated at Moorfields during the first 4 years of the war may be of general interest.

Of the 154 cases in which a magnetic foreign body was successfully removed from the eye during that period, the posterior route was used only twice, and of these, one eye became shrunken and siderotic, and the other required enucleation early from sepsis. Removal through the scleral wound of entry was effected in 2 . further cases, the resultant vision being reduced to $6 / 18$ from a localised cataract and 6/60 from macular striae respectively.,

In the remaining 150 cases, the anterior route was employed. In about 15 per cent. of these the foreign body lay in the anterior chamber, and in the remainder it was drawn thither by the Haab magnet (or in 3 cases only by the Mellinger), final removal being effected with the hand magnet through a keratome section. The vision was estimated when the eye condition could be considered static 6 months to 3 years later, except in the very few cases in which the patient was out of reach, when an earlier record had to be accepted.

- Received for publication, February 23, 1944. 